\title{
A chromosomal ars operon homologue of Pseudomonas aeruginosa confers increased resistance to arsenic and antimony in Escherichia coli
}

\author{
Jie Cai, + Kirsty Salmon and Michael S. DuBow \\ Author for correspondence: Michael S. DuBow. Tel: +1 514398 3926. Fax: +1 5143987052. \\ e-mail: msdubow@microimm.mcgill.ca
}

Department of Microbiology and Immunology, McGill University, 3775 University Street, Montreal, Quebec, Canada H3A 2B4

\begin{abstract}
Operons encoding homologous arsenic-resistance determinants (ars) have been discovered in bacterial plasmids from Gram-positive and Gram-negative organisms, as well as in the Escherichia coli chromosome. However, evidence for this arsenic-resistance determinant in the medically and environmentally important bacterial species Pseudomonas aeruginosa is conflicting. Here the identification of a P. aeruginosa chromosomal ars operon homologue via cloning and complementation of an $E$. coli ars mutant is reported. The $P$. aeruginosa chromosomal ars operon contains three potential ORFs encoding proteins with significant sequence similarity to those encoded by the arsR, ars $B$ and ars $C$ genes of the plasmid-based and $E$. coli chromosomal ars operons. The cloned $\boldsymbol{P}$. aeruginosa chromosomal ars operon confers augmented resistance to arsenic and antimony oxyanions in an $E$. coli ars $B$ mutant and in wild-type $P$. aeruginosa. Expression of the operon was induced by arsenite at the MRNA level. DNA sequences homologous with this operon were detected in some, but not all, species of the genus Pseudomonas, suggesting that its conservation follows their taxonomic-based evolution.
\end{abstract}

Keywords: arsenic resistance, Pseudomonas, chromosomal ars operon, evolutionary conservation

\section{INTRODUCTION}

Plasmid-based arsenic resistance in bacteria, encoded by the ars operons, has been known for many years (Kaur $\&$ Rosen, 1992). Five genes, transcribed as a single polycistronic $\mathrm{mRNA}$ in the order arsRDABC, have been found in Escherichia coli plasmids R773 and R46 (Owolabi \& Rosen, 1990; Bruhn et al., 1996). These ars operons confer resistance to arsenic and antimony oxyanions via an ATP-dependent efflux mechanism (Mobley \& Rosen, 1982). The first two genes, arsR and ars $D$, of the R773 ars operon encode two inducerdependent trans-acting repressors that control the basal and upper levels of operon expression, respectively (San

\footnotetext{
†Present address: Biochemistry Department, McGill University, 3655 Drummond Street, Montreal, Quebec, Canada H3G IY6.

Abbreviation: SD, Shine-Dalgarno sequence.

The EMBL accession number for the nucleotide sequence reported in this paper is AF010234.
}

Francisco et al., 1990; Wu \& Rosen, 1991, 1993a, b; Chen \& Rosen, 1997). True inducers for expression of the ars operon are trivalent, but not pentavalent, arsenic and antimony oxyanions (Wu \& Rosen, 1993b; Chen $\&$ Rosen, 1997). The ars $A B C$ genes encode the structural components of the arsenic pump (Chen et al., 1986). The ArsA protein is an arsenite- and antimonite-stimulated ATPase (Rosen et al., 1988) which forms a complex with the membrane-bound ArsB protein (Tisa \& Rosen, 1990; Wu et al., 1992), and actively exports arsenite and antimonite ions upon hydrolysis of ATP (Kaur \& Rosen, 1994a, b; Li et al., 1996). Interestingly, the ArsB protein of the R773 ars operon actively exports arsenite ions even in the absence of the ArsA ATPase (Dey \& Rosen, 1995), suggesting that both ATP and membrane potential can stimulate the function of the ArsB membrane pump. Consistent with this, the ars operons in the Gram-positive staphylococcal plasmids pI258 and pSX267 contain only ars $R$, ars $B$ and ars $C$ genes, lacking the $\operatorname{ars} A$ and $a r s D$ coding sequences. Nonetheless, these ars operons can actively export arsenite and antimony 
oxyanions using membrane potential, rather than cellular ATP, as an energy source (Ji \& Silver, 1992b; Rosenstein et al., 1992; Bröer et al., 1993). The ArsC protein is a small cytosolic protein that acts to reduce arsenate to arsenite (Gladysheva et al., 1994; Oden et al., 1994), which can then be extruded by the ArsAB complex.

The recently discovered E. coli chromosomal ars operon also contains three ORFs, arsRBC, structurally resembling the staphylococcal plasmid ars operons (Sofia et al., 1994; Diorio et al., 1995), and is functional in conferring increased resistance to arsenic (Carlin et al., 1995; Diorio et al., 1995). Moreover, an arsH gene is required for arsenic resistance in a Yersinia enterocolitica plasmid-located ars operon, although the function of the arsH product has not yet been elucidated (Neyt et al., 1997). In addition to resistance to arsenic and antimony oxyanions, the plasmid R773 ars operon can confer increased resistance to tellurite (Turner et al., 1992), a result not observed with the E. coli chromosomal ars operon (Cai \& DuBow, 1996), though whether this was due to the inability of tellurite to induce ars operon expression has yet to be determined. Nonetheless, significant sequence similarity between the $E$. coli chromosomal and the plasmid-encoded ars operons (Diorio et al., 1995) suggests a common ancestor. Since the E. coli chromosomal ars operon is the only functionally characterized chromosomally located ars operon in bacteria to date, it is important to identify and characterize others to better understand the evolutionary relationship among ars operons.

We have previously observed (via Southern blotting) that sequences hybridizing with the E. coli chromosomal ars operon are present in the chromosomes of many Gram-negative bacterial species, including the nonenteric bacterium Pseudomonas aeruginosa (Diorio et al., 1995). Although it has been reported that pUM310 of $P$. aeruginosa contains a genetic determinant that confers resistance to arsenic and antimony ions (Cervante \& Chávez, 1992), the evidence for a chromosomal ars operon in members of the genus Pseudomonas has been controversial (Carlin et al., 1995; Diorio et al., 1995). The aim of this study was to determine if an ars operon homologue exists in the chromosome of $P$. aeruginosa. Here we report the isolation, DNA sequencing, and $E$. coli ars operon complementation of a chromosomal ars operon of $P$. aeruginosa. It consists of three ORFs encoding protein products similar in sequence to those encoded by the ars $R$, ars $B$ and ars $C$ genes of known ars operons. The $P$. aeruginosa ars operon is functional in conferring increased resistance to arsenic and antimony oxyanions, as determined by complementation of an E. coli arsB mutant. Moreover, expression of this operon, as measured by RNA dot blots, is induced by sodium arsenite. Sequence analyses revealed that the $P$. aeruginosa chromosomal ars operon is evolutionarily related to plasmid-based ars operons from both Gram-negative and Gram-positive bacteria, and is conserved in the chromosomes of some, but not all, Pseudomonas species.

\section{METHODS}

Bacterial strains and plasmids. E. coli strain $40\left[\mathrm{~F}^{-} \Delta(\right.$ pro-lac $)$ rpsL trp] is an E. coli K-12 strain in which we isolated and sequenced the chromosomal ars operon (Diorio et al., 1995). $E$. coli strain LF20012 is derived from E. coli strain 40, but contains an ars $B:: \operatorname{lu} x A B$ chromosomal transcriptional gene fusion (Cai \& DuBow, 1996), and is thus genotypically arsB and hypersensitive to arsenic oxyanions (Cai \& DuBow, 1997). P. aeruginosa PAO1, Pseudomonas fluorescens, Pseudomonas stutzeri, Pseudomonas diminuta and Burkbolderia cepacia (formerly Pseudomonas cepacia) are all plasmid-free wild-type strains obtained from Dr G. Hegeman (Indiana University, USA). $P$. aeruginosa wild-type strain PAK was from Dr R. Ramphal (University of Florida, USA). E. coli cultures were grown in Luria-Bertani (LB) medium (Miller, 1972) at $37^{\circ} \mathrm{C}$. Cultures of Pseudomonas and Burkholderia species were grown initially in Brain Heart Infusion (BHI; Difco) broth and then in LB broth at $32{ }^{\circ} \mathrm{C}$, except $P$. fluorescens, which was grown at $25^{\circ} \mathrm{C} . \mathrm{pJC} 801\left(\mathrm{Ap}^{\mathrm{r}}\right)$ is a pBR322 derivative that contains the cloned $P$. aeruginosa chromosomal ars operon as a $3.4 \mathrm{~kb}$ EcoRV fragment. The same restriction fragment was also cloned into pTJS140 (Darzins \& Casadaban, 1989), yielding pKAS300 (described in the following sections). pUC119 and pUC118 (Vieira \& Messing, 1987; Sambrook et al., 1989) were used as vectors for subcloning and sequencing the $P$. aeruginosa chromosomal ars operon. Ampicillin and piperacillin were used at a final concentration of $40 \mu \mathrm{g} \mathrm{ml}^{-1}$ and $50 \mu \mathrm{g} \mathrm{ml}^{-1}$, respectively, when required.

Cloning and sequencing of the $\boldsymbol{P}$. aeruginosa chromosomal ars operon. All restriction enzymes and T4 DNA ligase used for cloning purposes were purchased from New England Biolabs and used following the supplier's instructions. Isolation of plasmid and genomic DNA, preparation of competent $E$. coli cells, and DNA transformation into $E$. coli cells were performed as described by Sambrook et al. (1989). Preparation of competent PAO1 cells was performed according to Farinha \& Kropinski (1990). Transformation of pTJS140 and pKAS300 into PAO1 was performed by electroporation. $\alpha-{ }^{32} \mathrm{P}$-labelling of DNA probes for Southern and RNA dot-blotting analyses was performed using the random priming method (Sambrook et al., 1989) with random hexanucleotide primers (Regional DNA Synthesis Laboratory, University of Calgary, Canada). Radioisotopes were purchased from Amersham Life Science.

To clone the $P$. aeruginosa strain PAO1 chromosomal ars operon homologue, restriction fragments approximately $3.4 \mathrm{~kb}$ in size, previously shown to hybridize to the E. coli chromosomal ars operon (Diorio et al., 1995), were purified from a $0.8 \%$ agarose gel of EcoRV-cleaved chromosomal DNA of $P$. aeruginosa strain PAO1 using the Geneclean II kit (Bio101), and ligated to EcoRV-linearized pBR322. The ligation mixture was transformed into $E$. coli strain JM105 (Yanisch-Perron et al., 1985). Recombinant transformants were selected for ampicillin resistance and screened for tetracycline sensitivity. To identify the plasmids containing the putative $P$. aeruginosa chromosomal ars operon homologue, clones from the recombinant transformants were lysed by the 'cracking' procedure (Barnes, 1977), subjected to $0.8 \%$ agarose gel electrophoresis, transferred to a Hybond- $\mathrm{N}$ nylon membrane (Amersham Life Science) and hybridized (Diorio et al., 1995) to a ${ }^{32} \mathrm{P}$-labelled E. coli chromosomal arsB probe, isolated as a $658 \mathrm{bp} S c a \mathrm{I}-\mathrm{Punll}$ restriction fragment from pJC701 (Cai \& DuBow, 1996). Following autoradiography, plasmid DNA was isolated from recombinant clones with enhanced hybridization signals (Sambrook et al., 1989), 
and used to transform E. coli LF20012 (arsB mutant). The transformants were screened for enhanced resistance to sodium arsenite (when compared with E. coli LF20012 containing pBR322) by replica plating them on LB agar containing increasing concentrations of sodium arsenite $(0$, $20,100,200,400$ and $800 \mu \mathrm{g} \mathrm{As} \mathrm{ml}^{-1}$ ). One of the clones showing enhanced arsenic resistance was isolated, and its recombinant plasmid, containing a $3.4 \mathrm{~kb}$ EcoRV $P$. aeruginosa chromosomal DNA fragment, was named pJC801. The DNA fragment cloned in pJC801 was subcloned in the

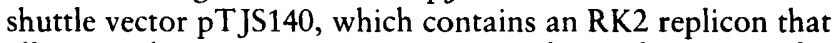
allows replication in $P$. aeruginosa and a ColE1 origin for replication in E. coli. The recombinant plasmid from E. coli, called pKAS300, was used to transform $P$. aeruginosa strain PAO1.

Fragments of the $P$. aeruginosa chromosomal DNA in pJC801 were further subcloned into pUC119 or pUC118 (Sambrook $e t$ al., 1989). Double- or single-stranded DNA templates for sequencing analysis were prepared using the QIAprep Spin Mini Prep kit (Qiagen), or following the method of Vieira \& Messing (1987). DNA sequencing of both strands of these subclones was performed manually by the dideoxy DNA sequencing method using the ISOTHERM sequencing kit (Epicentre Technologies), and by automatic sequencing (Core Facilities for Protein/DNA Chemistry, Biochemistry Department, Queen's University, Kingston, Ontario, Canada).

\section{Complementation assays in an $E$. coli arsB strain and determination of the arsenic-resistance phenotype and MICs in wild-type $E$. coli and P. aeruginosa. The plasmid pJC801 (and the parental plasmid pBR322) were used to transform the E. coli ars B mutant strain LF20012 (arsB::luxAB) (Cai \& DuBow, 1996), as well as its parent, E. coli 40 . The resistance profile to arsenic and antimony salts of all four transformants was determined by their ability to grow in LB broth containing increasing amounts of arsenic or antimony oxyanions as follows. Overnight cultures of each sample were diluted 100 - fold in LB broth containing the appropriate antibiotics and increasing concentrations of sodium arsenite, sodium arsenate and potassium antimony tartrate hemihydrate. The cultures were incubated for $6 \mathrm{~h}$ at $37^{\circ} \mathrm{C}$, and the $\mathrm{OD}_{600}$ of each sample (in triplicate) was determined. The resistance profile of each strain to arsenic or antimony oxyanions was expressed as the percentage $\mathrm{OD}_{600}$ compared with the control culture with no added arsenic or antimony oxyanions calculated (Cai \& DuBow, 1997) as a function of the elemental concentrations of arsenic or antimony in the compounds used.}

The resistance phenotype of the $P$. aeruginosa PAO1 strain with or without the cloned $P$. aeruginosa chromosomal ars operon homologue was also determined. Since $P$. aeruginosa PAO1 is normally somewhat mucoid, cell growth cannot be measured accurately by its turbidity in liquid medium (not shown). Therefore, sensitivity of these strains to arsenic/ antimony oxyanions was measured by dilution (to 100 -fold) of an overnight culture of $P$. aeruginosa PAO1 into LB broth, growing to an $\mathrm{OD}_{600}$ of 0.2 in a $32^{\circ} \mathrm{C}$ air shaker. Aliquots of diluted exponentially growing cells $\left(100 \mu \mathrm{l}\right.$ of the $10^{-6}$ dilution per plate) were then plated on LB agar containing increasing concentrations of arsenic and antimony salts. The c.f.u. of each strain were determined after $24 \mathrm{~h}$ growth of the cells at $32{ }^{\circ} \mathrm{C}$. Each assay was performed in triplicate. The mean number of c.f.u. of each strain in the absence of added chemicals was defined as $100 \%$ survival. The percentage survival of each strain in the presence of specific concentrations of added chemicals was expressed as its mean c.f.u. value with the oxyanions present, divided by its c.f.u. value in the absence of added chemicals.
The MICs of each oxyanion for the wild-type Pseudomonas strains were measured after diluting the overnight culture $(1: 100)$ in LB broth containing increasing concentrations of arsenite, arsenate and antimonite, and determined as the minimum concentrations that resulted in no increase in cell density $\left(\mathrm{OD}_{600}\right)$ after $6 \mathrm{~h}$ incubation in an air shaker.

Southern blotting analysis. Genomic DNAs $(10 \mu \mathrm{g})$ were cleaved with EcoRV and PstI, fractionated by $0.8 \%$ agarose gel electrophoresis and transferred to a Hybond-N membrane following denaturing and neutralization (Sambrook et al., 1989). The subsequent prehybridization and hybridization with a ${ }^{32} \mathrm{P}$-radiolabelled probe were performed as previously described (Autexier \& DuBow, 1992; Diorio et al., 1995). Briefly, the membrane was incubated with prehybridization solution [ $5 \times$ SSC, $5 \times$ Denhardt's solution (Sambrook et al., 1989), $50 \mu \mathrm{g}$ calf thymus DNA ml ${ }^{-1}, 50 \%$ (v/v) deionized formamide] for $2 \mathrm{~h}$ at $40^{\circ} \mathrm{C}$, and then with hybridization solution [ $5 \times$ SSC, $1 \times$ Denhardt's solution, $50 \mu \mathrm{g}$ calf thymus DNA ml ${ }^{-1}, 50 \%(\mathrm{v} / \mathrm{v})$ deionized formamide, $0 \cdot 3 \%(\mathrm{w} / \mathrm{v}) \mathrm{SDS}$, $25 \mu \mathrm{M}$ ATP and $1 \mathrm{mM}$ EDTA, pH 8.0] containing 2$4 \times 10^{5}$ c.p.m. labelled probe for $16-18 \mathrm{~h}$ at $39^{\circ} \mathrm{C}$. The probe was isolated from pJC801 as a $1 \cdot 14 \mathrm{~kb} B a m \mathrm{HI}-\mathrm{Sp} h \mathrm{I}$ fragment containing the second half of ars $R$ and the first two-thirds of arsB. After hybridization, the membrane was washed (in a solution containing $0.2 \%, \mathrm{w} / \mathrm{v}, \mathrm{SDS}$ and $0.5 \times \mathrm{SSC}$ ) three times for $30 \mathrm{~min}$ at room temperature, and exposed to Kodak XAR-5 film (Eastman Kodak), using Dupont Cronex intensifying screens.

Preparation of total cellular RNA of P. aeruginosa PAO1 and dot-blotting analysis. Cultures of $P$. aeruginosa strain PAO1 were grown in $\mathrm{LB}$ broth at $32^{\circ} \mathrm{C}$ in a shaking incubator until mid-exponential phase $\left(\mathrm{OD}_{600}=0 \cdot 5-0 \cdot 6\right)$, and then sodium arsenite was added to a final concentration of $0 \cdot 1 \mu \mathrm{g} \mathrm{As} \mathrm{ml}^{-1}$. Choice of this concentration was based on our previous observations for the E. coli ars operon (Cai \& DuBow, 1996). Samples were removed at $0,15,30$ and $60 \mathrm{~min}$ after arsenic addition and kept on ice until all samples were ready. Total cellular RNA was isolated using the RNaid Plus kit (Bio101), following the manufacturer's instructions. The RNA concentrations of each sample were spectrophotometrically determined using a UV-1201 spectrophotometer (Shimadzu Scientific Instruments). Different amounts $(5,2.5$ and $1 \mu \mathrm{g}$ per slot) of total cellular RNA from arsenite-exposed and unexposed $P$. aeruginosa were loaded onto a $\mathrm{Hybond}-\mathrm{N}$ nylon membrane (Amersham Life Science) in a Bio-Rad dot-blot apparatus as described by Sambrook et al. (1989). The RNA was fixed to the membrane by microwave exposure for $2.5 \mathrm{~min}$ and hybridized to the same probe as used for Southern blotting. Prehybridization and hybridization reactions were performed as described by Cai \& DuBow (1996), and the membrane was washed four times for $15 \mathrm{~min}$ at $65^{\circ} \mathrm{C}$ in a solution containing $40 \mathrm{mM} \mathrm{Na} \mathrm{PO}_{4}, \mathrm{pH} 7 \cdot 2,1 \mathrm{mM}$ EDTA, $1 \%(w / v)$ SDS, and exposed to Kodak XAR-5 film.

\section{RESULTS}

\section{Cloning of an ars operon homologue of $P$. aeruginosa}

Based upon our previous observation that an approximately $3.4 \mathrm{~kb} E c o \mathrm{RV}$ DNA fragment of $P$. aeruginosa contained sequences homologous to the E. coli chromosomal ars operon, and thus a potential chromosomal ars operon homologue (Diorio et al., 1995), EcoRV fragments of this size were isolated and cloned in pBR322. $E$. coli transformants were screened to detect sequences 
Table 1. Percentage identity (similarity) of $P$. aeruginosa ArsR, B and C polypeptides to amino acid sequences deduced from known ars genes

\begin{tabular}{|lccc|}
\hline ars gene & ArsR & ArsB & ArsC \\
\hline E. coli & $44.4(55.5)$ & $69.3(84.9)$ & $10.6(27.6)$ \\
R773 & $43.6(59.8)$ & $69.3(85.6)$ & $12.8(24.1)$ \\
IncN R46 & $43.6(57.3)$ & $69.9(85.5)$ & $12.1(23.6)$ \\
Tn2505 & $42.7(56.4)$ & $68.9(83.3)$ & $13.5(29 \cdot 1)$ \\
pI258 & $28.8(49.0)$ & $51.7(72.7)$ & $29.0(45.8)$ \\
pSX267 & $27.9(46.2)$ & $51.4(72.6)$ & $29.0(45.8)$ \\
\hline
\end{tabular}

hybridizing with an $E$. coli ars $B$ probe. Four putative clones were confirmed by a complementation assay, and were isolated due to their ability, in transformants of an E. coli arsB mutant host, to confer high levels of resistance to arsenite (i.e. the ability to allow growth on solid media containing $400 \mu \mathrm{g} \mathrm{As} \mathrm{ml}^{-1}$ as sodium arsenite; not shown). Based upon restriction enzyme mapping, these four plasmids, designated pJC801 through pJC804, contained an identical DNA insert (data not shown). Therefore, pJC801 was chosen for further studies.

\section{DNA and predicted amino acid sequence analyses}

Subcloning and DNA sequencing analyses showed that the cloned $P$. aeruginosa PAO1 chromosomal DNA fragment contained three ORFs encoding proteins with significant sequence similarities to the ArsR, ArsB and ArsC polypeptides of the $E$. coli chromosomal and plasmid-encoded ars operons. Comparison of the amino acid sequences of the $P$. aeruginosa ars operon with other known ars operons, using the method of Myers \& Miller (1988), revealed striking similarities, summarized in Table 1. In the case of ArsR, the amino acid sequence identity between the $P$. aeruginosa chromosomal ars operon and other ars operons ranged from 28 to $44 \%$ (Table 1). In particular, the putative metal-binding box (ELCVCDL) and the DNA-binding helix-turn-helix motif identified in the ArsR proteins of other ars operons and known to be critical in arsenic-dependent regulation of operon expression, were also conserved in the ArsR sequence of the $P$. aeruginosa chromosomal ars operon (Fig. 1). These results suggest that the $P$. aeruginosa chromosomal ars operon is a new member of the arsenic efflux system family, and may be regulated in a manner similar to that used by other known ars operons. The highest sequence identity, ranging from 51 to $70 \%$, was found between the ArsB protein of the $P$. aeruginosa chromosomal ars operon and that of other known ars operons (Table 1). Multiple alignments (Higgins \& Sharp, 1988, 1989) of the P. aeruginosa ArsB sequence with the ArsB proteins of the ars operons listed in Table 1 revealed an overall amino acid identity (similarity) of $45.3 \%(78.8 \%)$ (data not shown). Hydrophobicity analyses, using the method of Eisenberg et al. (1984), indicate that the ars $B$ gene product is a hydrophobic protein with 12 putative membrane-associated helices,
ArsR PAO1

ArsR E. coll ArsR R773 ArsR R46

ArsR Tn2502

Ars 1258

ArsR pS $\times 267$

ArsR PAO1

ArsR E. coli

ArsR R773

ArsR R46

ArsR Tn2502

ArsR pl258

ArsR pSX267

ArsR PAO1

ArsR E. coli

ArsR R773

ArsR R46

ArsR Tn2502

ArsR pl258

ArsR pSX267

ArsR PAO1

ArsR E. coli

ArsR R773

ArsR R46

ArsR Tn2502

ArsR pl258

ArsR pSX267

ArsR PAO1

ArsR E. coli

ArsR R773

ArsR R46

ArsR Tn2502

ArsR p1258

ArsR pSX267
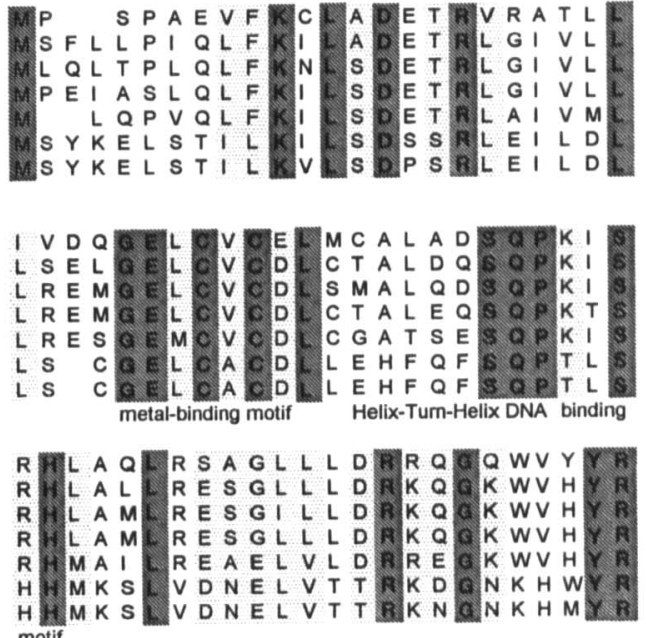

motif

NPALPAWI HEVLOVTLRGQPATG

SPHI PA WAAKII DEAWRCEQEKV

SPHI PS WAAQI I E QAWLS QQDDV

S P H I P S WA A L I E QAWL S Q D D V

S P H M A WA AETITTS WHCC GKM

NHA I L D D I I QNLN

NHEFLDYINQNLD

Fig. 1. Multiple alignments of amino acid sequences of the ArsR proteins from various ars operons using the CLUSTAL computer program (PC/Gene software, IntelliGenetics). Identical amino acid residues are indicated in hatched areas; similar amino acid residues are indicated in dotted areas. The potential metal-binding and DNA-binding motifs are marked.

consistent with its potential function as a membrane transporter and with the structures of other known ArsB proteins. It is interesting to note that the ArsR and ArsB proteins of the $P$. aeruginosa chromosomal ars operon show close similarity to homologues from the ars operons of Gram-negative bacteria. By contrast, the ArsC protein is more similar to the ArsC proteins of Gram-positive ars operons (Table 1). Analysis of the codon usage of the $P$. aeruginosa ars operon indicated that the $P$. aeruginosa ars genes preferentially contain a cytosine at the third nucleotide of their amino acid codon position when compared with those of the E. coli chromosomal ars operon, consistent with the codon usage of many other chromosomal genes of $P$. aeruginosa (West \& Iglewski, 1988).

\section{The cloned $\boldsymbol{P}$. aeruginosa chromosomal ars operon confers increased arsenite and antimonite resistance in both $E$. coli and $P$. aeruginosa}

To examine its role in arsenic resistance, the cloned $P$. aeruginosa chromosomal ars operon was introduced into E. coli and P. aeruginosa PAO1. In E. coli, pJC801 was used to transform the arsB mutant strain LF20012 

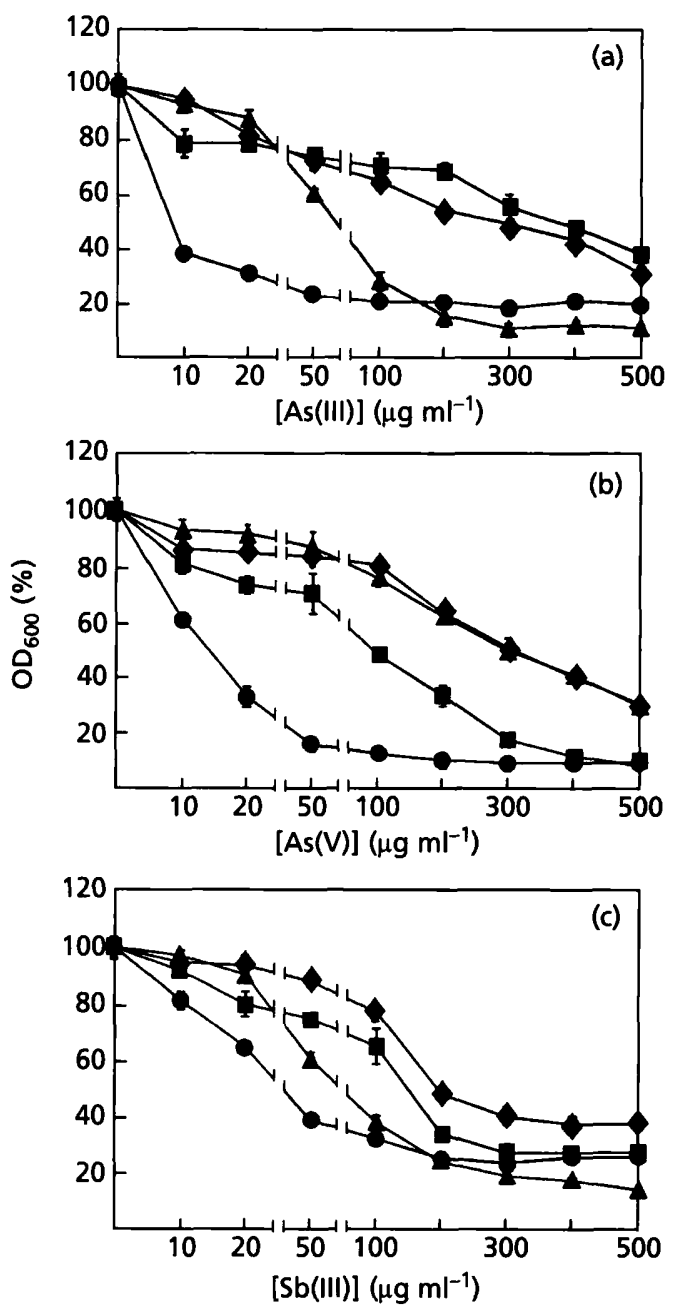

Fig. 2. Growth of plasmid-containing $E$. coli strains in the presence of increasing amounts of arsenic and antimony oxyanions. Overnight cultures were diluted 100-fold in LB broth containing the appropriate antibiotics and increasing amounts of arsenic or antimony salts. Cellular growth was measured $6 \mathrm{~h}$ after addition of chemicals and expressed as percentage $O D_{600}$ versus elemental concentrations of arsenic (antimony) added to the growth media (see Methods). Standard deviations are represented by error bars. $\triangle, E$. coli 40(pBR322); $\diamond, E$. coli 40(pJC801); O, LF20012(pBR322); 口, LF20012(pJC801).

(Cai \& DuBow, 1996), and the parental strain $40\left(\right.$ ars $\left.^{+}\right)$. As controls, both $E$. coli strains were transformed with pBR322. Cultures from these four strains were analysed for their ability to grow on increasing concentrations of arsenite, arsenate and antimonite ions (Fig. 2). Introduction of pJC801 into $E$. coli conferred an increase in arsenite resistance in both ars $^{+}$and arsB strains in a manner similar to that observed when the E. coli chromosomal ars operon is cloned in a multicopy plasmid (Diorio et al., 1995) (Fig. 2a). A similar increase in antimonite resistance was also observed (Fig. 2c). $P$. aeruginosa strain PAO1, containing the cloned chromosomal ars operon on pKAS300, also showed increased resistance to all three oxyanions when compared to the strain containing the vector plasmid only (Fig. 3). These
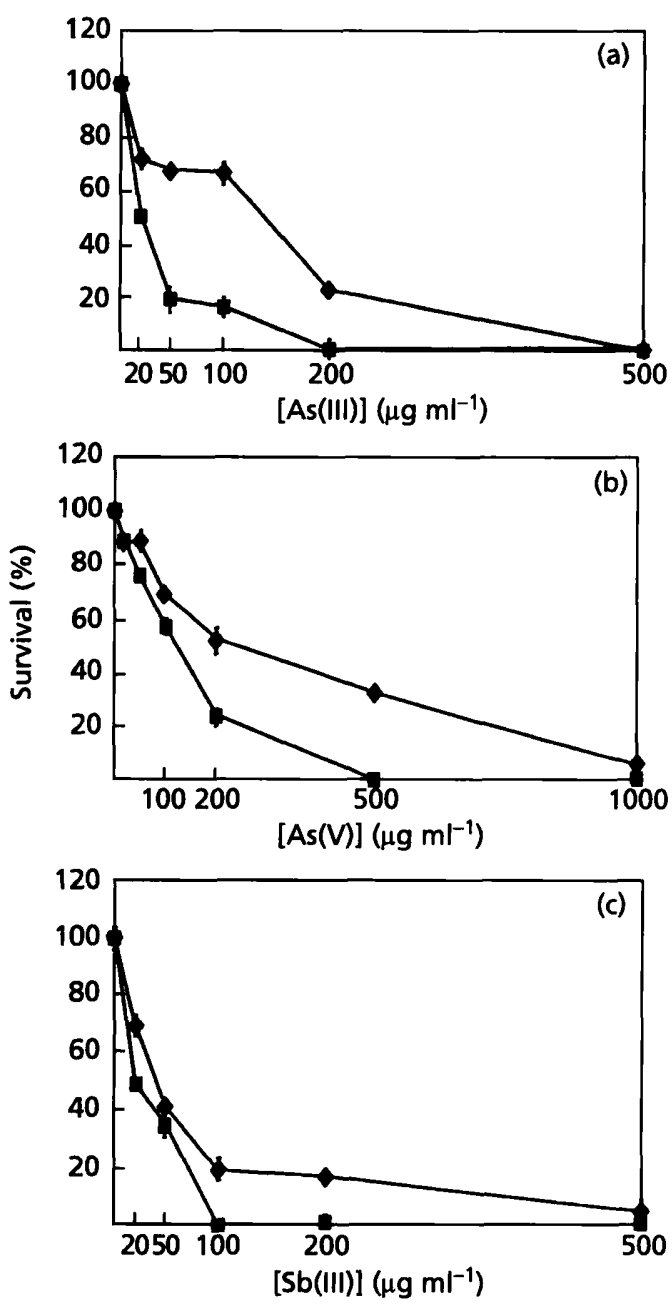

Fig. 3. Resistance profile of $P$. aeruginosa strains containing pKAS300 or PTJS140 to arsenite (a), arsenate (b) and antimonite (c). A $10^{-6}$ dilution of an exponentially growing culture of each strain was spread $(100 \mu \mathrm{l}$ per plate) onto LB agar plates containing $0,20,50,100,200,500$ and $1000 \mu \mathrm{g}$ arsenic or antimony $\mathrm{ml}^{-1}$ as sodium arsenite, sodium arsenate or potassium antimony tartrate, respectively. The percentage survival of each sample was determined as described in Methods. Standard deviations are represented by error bars. $\square$, PAO1(pTJS140); $\diamond$, PAO1(pKAS300).

results suggest that the $P$. aeruginosa ars operon not only increased resistance to all three oxyanions in $P$. aeruginosa when cloned in a high-copy-number plasmid, but also complemented an $E$. coli arsB mutant and augmented wild-type $E$. coli resistance to arsenite and antimonite ions. Although the cloned $P$. aeruginosa ars operon increased arsenate resistance in the $E$. coli ars $B$ mutant as well as in P. aeruginosa PAO1, it did not show any detectable enhancement of arsenate resistance in wild-type E. coli (Fig. 2b).

\section{Transcription of the $P$. aeruginosa chromosomal ars operon is inducible by sodium arsenite}

To determine if arsenic-regulated expression of the $P$. aeruginosa chromosomal ars operon occurs at the 
(a)

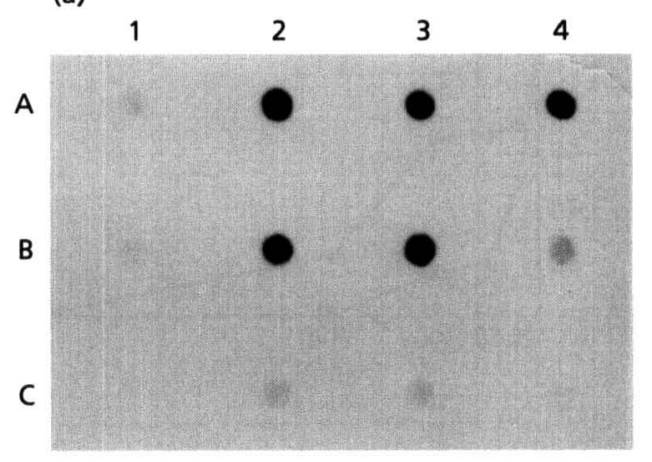

(b)

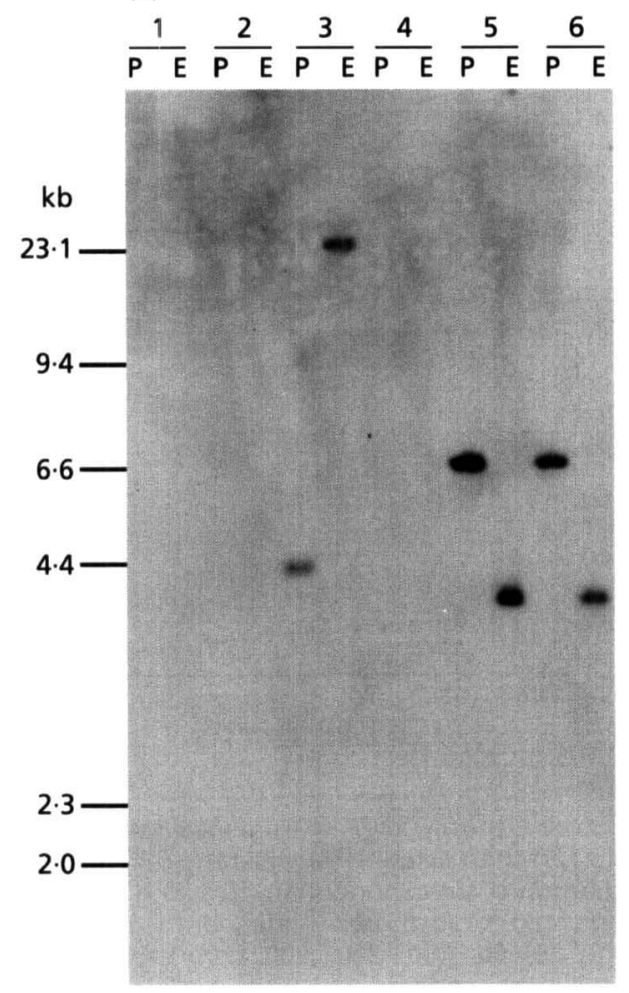

Fig. 4. (a) Dot-blot hybridization of total cellular RNA to a ${ }^{32} \mathrm{p}$. labelled $P$. aeruginosa chromosomal ars operon probe (see Methods). Lanes: 1, RNA from unexposed cultures; 2-4, RNAs from cells incubated with sodium arsenite (at a final concentration of $0.1 \mathrm{\mu g}$ As $\mathrm{ml}^{-1}$ ) for 15,30 and $60 \mathrm{~min}$, respectively. Samples in rows A, B and C are loaded with 5, 2.5 and $1 \mu \mathrm{g}$ RNA per slot, respectively. (b) Southern hybridization of genomic DNA from various bacterial species to a $32 \mathrm{p}$-labelled $P$. aeruginosa chromosomal ars operon (see Methods). The numbers on top indicate different bacterial strains: $1, B$. cepacia; 2, P. diminuta; 3, P. fluorescens; 4 , P. stutzeri; $5, P$. aeruginosa PAK; $6, P$. aeruginosa PAO1. The letters on top indicate the restriction enzymes used to digest the genomic DNAs: P, Pstl; E, EcoRV. The numbers on the left side are the positions migrated by DNA size markers during electrophoresis.

transcriptional level, equal amounts of total cellular RNA, isolated from sodium arsenite-exposed (for 15, 30 and $60 \mathrm{~min}$, respectively) and unexposed cells, were loaded onto a nylon membrane, fixed, dried and hybridized to a ${ }^{32} \mathrm{P}$-labelled DNA fragment containing
Table 2. MICs of arsenic and antimony oxyanions $\left(\mu \mathrm{g} \mathrm{ml}^{-1}\right)$

\begin{tabular}{|lrrr|}
\hline Strain & As(III) & As $(\mathbf{V})$ & Sb(III) \\
\hline P. aeruginosa & 200 & 800 & 75 \\
P. fluorescens & 200 & $>2000$ & $<25$ \\
P. diminuta & 75 & $>2000$ & $<25$ \\
P. stutzeri & 75 & $>2000$ & 50 \\
B. cepacia & 300 & $>2000$ & 50 \\
\hline
\end{tabular}

the $P$. aeruginosa chromosomal ars genes. The autoradiograph (Fig. 4a) showed a dramatic increase in hybridization in the arsenite-exposed RNA samples when compared to the unexposed sample, suggesting that transcription of the $P$. aeruginosa chromosomal ars operon is inducible by arsenic oxyanions. Quantification analysis using the Image Quant program (Molecular Dynamics) revealed at least a 15 -fold increase in arsspecific RNA upon addition of $0 \cdot 1 \mu \mathrm{g} \mathrm{As} \mathrm{m}{ }^{-1}$ (as sodium arsenite) for $15 \mathrm{~min}$, indicating that ars $\mathrm{mRNA}$ is rapidly induced by arsenite. This level of increase is in a similar range with that of the E. coli ars operon, as determined by an arsB : : lacZ gene fusion expression assay (Diorio et al., 1995). Moreover, a decrease in the intensity of the ars-specific RNA was observed at $60 \mathrm{~min}$ post-exposure (Fig. 4a), consistent with previous observations for the E. coli chromosomal and the plasmid R773 ars operons (Cai \& DuBow, 1996; Owolabi \& Rosen, 1990).

\section{The $P$. aeruginosa ars operon is conserved in the chromosomes of other Pseudomonas species}

Southern blotting analysis of chromosomal DNAs from other Pseudomonas species using a ${ }^{32} \mathrm{P}$-labelled probe containing part of the $P$. aeruginosa chromosomal ars operon (Fig. 4b) detected hybridizing sequences in the chromosomes of $P$. aeruginosa strain PAK, another major wild-type strain of $P$. aeruginosa (Minamishima et al., 1968; Takeya \& Amako, 1966), and of $P$. fluorescens. However, no hybridizing sequences were found in the chromosomal DNAs of $P$. stutzeri, $P$. diminuta and $B$. cepacia under the conditions used, suggesting that the $P$. aeruginosa chromosomal ars operon is conserved, at the DNA level, in some, but not all, Pseudomonas species.

To determine whether the conservation of the ars operon is related to the intrinsic level of resistance to these toxic oxyanions, the MICs of arsenic and antimony oxyanions for each of the plasmid-free wild-type Pseudomonas species were determined (Table 2). The intrinsic resistance to arsenite appears to be related to the presence of the ars operon in the Pseudomonas species examined, as $P$. aeruginosa and $P$. fluorescens were found to have higher MICs than the two species $(P$. diminuta and $P$. stutzeri) that do not have any detectable ars-homologous sequences. However, the conservation of the ars operon does not directly correlate with cellular resistance to arsenate and antimonite oxyanions. Moreover, $B$. 
cepacia showed the highest resistance to arsenite whilst displaying no detectable homology to the ars operon. These observations support the existence of other mechanisms against arsenic toxicity (Huang et al., 1993; Huckle et al., 1993).

\section{DISCUSSION}

The $P$. aeruginosa chromosomal ars operon is the second functionally characterized chromosomally located ars operon of bacteria identified so far. The significant sequence similarities shared by its three ORFs with arsR, ars $B$ and $\operatorname{ars} C$ of other known ars operons, and its ability to complement an $E$. coli ars mutant, confirmed its molecular identity as a member of the ars family. Multiple alignments of the amino acid sequences of the putative P. aeruginosa ArsR with the ArsR proteins of other known ars operons, using the CLUSTAL program (Higgins \& Sharp, 1988, 1989), revealed the conservation of the putative metal-binding motif and DNAbinding helix-turn-helix motif. This suggests that the $P$. aeruginosa chromosomal ars operon may be regulated in a similar manner to other known ars operons, and we found that expression of the operon was arsenic/ antimony-inducible at the transcriptional level. The fold induction is in a similar range to that of the $E$. coli ars operon. Among the three ars polypeptides, the putative ArsB protein of the $P$. aeruginosa chromosomal ars operon is the most conserved, consistent with what has been observed with other known ars operons (Silver $e t$ al., 1993; Diorio et al., 1995; Silver, 1996). Both the ArsR and ArsB proteins share greater homology with their respective proteins from Gram-negative ars operons, which is not unexpected since $P$. aeruginosa is a Gram-negative bacterial species. Surprisingly, the putative Ars $C$ protein of the $P$. aeruginosa chromosomal ars operon shares greater homology with the ArsC polypeptides of Gram-positive ars operons than with those of ars operons from Gram-negative bacteria.

A functional analysis of the $P$. aeruginosa chromosomal ars operon in $P$. aeruginos $a$ and $E$. coli suggests that, like its E. coli counterpart, it can provide enhanced resistance to arsenic and antimony oxyanions in both bacterial species when cloned in a multicopy plasmid (Carlin et al., 1995; Diorio et al., 1995). However, when the $P$. aeruginosa chromosomal ars operon was introduced into wild-type $E$. coli, only increased resistance to arsenite and antimonite was observed, and no detectable increase was found in arsenate resistance. This is in contrast to our observation that the $E$. coli chromosomal ars operon cloned in a multicopy plasmid and introduced into wild-type $E$. coli increased resistance to both arsenite and arsenate ions (Diorio et al., 1995). The lack of increased arsenate resistance could be due to several reasons. First, the MIC assay suggests that the intrinsic level of arsenate resistance in $P$. aeruginosa PAO1 is much lower than other Pseudomonas strains and species tested. Secondly, previous studies have shown that the Gram-positive ArsC protein of the Staphylococcus aureus plasmid pI258 ars operon requires thioredoxin and thioredoxin reductase for proper function ( Ji \&

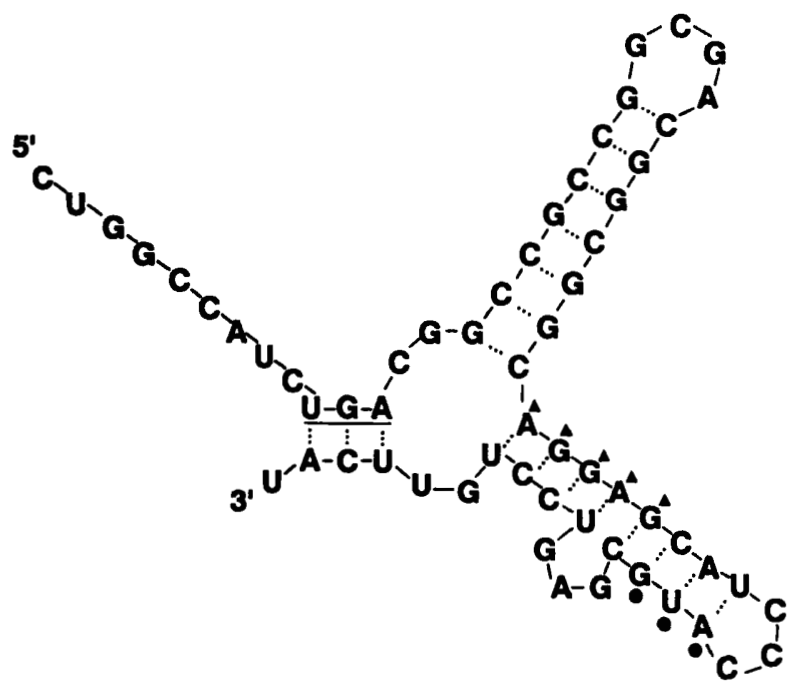

Fig. 5. Sequence and prediction of a potential mRNA secondary structure in the intergenic region between arsB and ars $C$ of the $P$. aeruginosa chromosomal ars operon. The termination codon UGA of the arsB gene is underlined. The putative ribosomebinding sequence (SD) is marked with triangles. The translation initiation codon AUG of the arsC gene is marked with circles. The free energy of formation of the indicated secondary structure is $-29.3 \mathrm{kcal} \mathrm{mol}^{-1}\left(-123 \mathrm{~kJ} \mathrm{~mol}^{-1}\right)$, as estimated by the Hibio DNASIS program (Hitachi Software Engineering).

Silver, 1992a ; Ji et al., 1994), whereas the activity of the Gram-negative ArsC protein from the E. coli plasmid $\mathrm{R} 773$ ars operon requires glutathione and glutathione reductase (Oden et al., 1994). As the ArsC protein is required for resistance to arsenate oxyanions, the physiological differences between $E$. coli and $P$. aeruginosa may contribute to suboptimal functioning of the $P$. aeruginosa ArsC protein in the heterologous cells. In addition, the closer relationship of ArsC to Grampositive ars operons may result in the ars $C$ gene of $P$. aeruginosa not being well expressed in $E$. coli. Analysis of the DNA sequence of the $P$. aeruginosa chromosomal ars operon revealed a larger intergenic region between the arsB and ars $C$ genes $(26 \mathrm{bp})$ than that found in other known ars operons (e.g. $12 \mathrm{bp}$ in the $E$. coli chromosomal and plasmid R773 ars operons). It is possible that this large intergenic region may negatively affect expression of the ars $C$ gene in $E$. coli, as previous studies have shown an optimal aligned spacing of $5 \mathrm{nt}$ between the Shine-Dalgarno (SD) sequence and the translationinitiation codon for ribosomes to efficiently translate mRNAs in E. coli (Chen et al., 1994). Secondary structures involving the SD sequence and internal complementary sequences inhibit translation initiation (Chang et al., 1995), and predictions of the $P$. aeruginosa chromosomal ars mRNA in the intergenic region between the ars $B$ and ars $C$ cistrons show a potential secondary structure (Fig. 5) involving both the putative SD sequence and the initiation codon of ArsC. Formation of this secondary structure in the ars mRNA could hinder the binding of ribosomes to the SD sequence and inhibit the initiation of translation. None- 
theless, in an ars mutant background, a low level of ArsC expression may still lead to significant reduction of arsenate to arsenite, and result in the observed higher level of arsenate resistance in E. coli LF20012(pJC801) than in strain LF20012(pBR322). This low-level expression (or activity) of ArsC from pJC801 may have been obscured by the fully induced endogenous $E$. coli chromosomal ars $\mathrm{C}$ gene, leading to no observable increase of arsenate resistance in wild-type $E$. coli.

It has been shown that both the $E$. coli chromosomal and plasmid R773 ars operons are transcribed upon arsenic exposure, and that the transcripts are processed within 1 h (Cai \& DuBow, 1996; Owolabi \& Rosen, 1990). Evidence also indicated that overexpression of the E. coli ArsR or ArsBC proteins is toxic to the cells (Cai \& DuBow, 1996). Therefore, a control on the upper level of ars mRNA (provided, where present, by the ArsD protein) may protect the cells from ars-polypeptide-induced toxicity. In the present study, the $P$. aeruginosa chromosomal ars operon is found to be transcribed upon cellular exposure to subinhibitory levels of arsenite ions, and the level of ars-specific mRNA decreases with prolonged induction time, consistent with what has been observed for other ars operons, and a potential mechanism to repress further expression of the ars-encoded proteins. Southern blotting revealed ars-homologous sequences in $P$. aeruginosa strain PAK and in P. fluorescens, but not in P. stutzeri, $P$. diminuta and $B$. cepacia, a species formerly classified as $P$. cepacia. The latter three species are more distantly related to $P$. aeruginosa than $P$. fluorescens. It is interesting to note that this conservation pattern was not directly reflected in the intrinsic level of resistance to these toxic oxyanions (i.e. MICs) in these bacterial species, suggesting the existence of other cellular mechanisms involved in arsenic resistance in these micro-organisms. Further genomic sequencing and identification of chromosomal ars operons in other bacteria will shed light on the evolution of this highly conserved and important bacterial operon. Moreover, recent results have shown this type of arsenic resistance mechanism in mammals (Wang \& Rossman, 1993; Wang et al., 1994, 1996), supporting the notion that the protective function of ars operons has been strongly conserved in the course of evolution.

\section{ACKNOWLEDGEMENTS}

We sincerely thank Caroline Diorio for her help and suggestions during the course of this research and the reviewers for their comments and suggestions. The work was supported by a grant from the Natural Sciences and Engineering Research Council (NSERC) of Canada (OGP0003222).

\section{REFERENCES}

Autexier, C. \& DuBow, M. S. (1992). The Escherichia coli Mu/D108 phage ner homologue gene $(n l p)$ is transcribed and evolutionarily conserved among the Enterobacteriaceae. Gene 114, 13-18.
Barnes, W. M. (1977). Plasmid detection and sizing in single colony lysates. Science 195, 393-394.

Bröer, S., Ji, G., Bröer, A. \& Silver, S. (1993). Arsenic efflux governed by the arsenic resistance determinant of Staphylococcus aureus plasmid pI258. J Bacteriol 175, 3480-3485.

Bruhn, D. F., Li, J., Silver, S., Roverto, F. \& Rosen, B. P. (1996). The arsenical resistance operon of IncN plasmid R46. FEMS Microbiol Lett 139, 149-153.

Cai, J. \& DuBow, M. S. (1996). Expression of the Escherichia coli chromosomal ars operon. Can J Microbiol 42, 662-671.

Cai, J. \& DuBow, M. S. (1997). Use of a luminescent bacterial biosensor for biomonitoring and characterization of arsenic toxicity in chromated copper arsenate. Biodegradation 8, 97-103.

Carlin, A., Shi, W., Dey, S. \& Rosen, B. P. (1995). The ars operon of Escherichia coli confers arsenical and antimonial resistance. $J$ Bacteriol 177, 981-986.

Cervante, C. \& Chávez, J. (1992). Plasmid-determined resistance to arsenic and antimony in Pseudomonas aeruginosa. Antonie Leeuwenboek 61, 333-337.

Chang, J. T., Green, C. B.-R. \& Wolf, R. E., Jr (1995). Inhibition of translation on Escherichia coli gnd mRNA by formation of a long-range secondary structure involving the ribosome binding site and the internal complementary sequence. J Bacteriol 177 , 6560-6567.

Chen, Y. \& Rosen, B. P. (1997). Metalloregulatory properties of the ArsD repressor. J Biol Chem 272, 14257-14262.

Chen, C. M., Misra, T. K., Silver, S. \& Rosen, B. P. (1986). Nucleotide sequence of the structural genes for an anion pump: the plasmid-encoded arsenical resistance operon. I Biol Chem 261, 15030-15038.

Chen, H., Bjerknes, M., Kumar, R. \& Jay, E. (1994). Determination of the optimal aligned spacing between the Shine-Dalgarno sequence and the translation initiation codon of Escherichia coli mRNAs. Nucleic Acids Res 22, 4953-4957.

Darzins, A. \& Casadaban, M. J. (1989). Mini-D3112 bacteriophage transposable elements for genetic analysis of Pseudomonas aeruginosa. J Bacteriol 171, 3909-3916.

Dey, S. \& Rosen, B. P. (1995). Dual mode of energy coupling by the oxyanion translocating ArsB protein. J Bacteriol 177, 385-389.

Diorio, C., Cai, J., Marmor, J., Shinder, R. \& DuBow, M. S. (1995). An Escherichia coli chromosomal ars operon homolog is functional in arsenic detoxification and conserved in Gram-negative bacteria. J Bacteriol 177, 2050-2056.

Eisenberg, D., Schwarz, E., Komaromy, M. \& Wall, R. (1984). Analysis of membrane and surface protein sequences with the hydrophobic moment plot. J Mol Biol 179, 125-142.

Farinha, M. A. \& Kropinski, A. M. (1990). High efficiency electroporation of Pseudomonas aeruginosa using frozen cell suspensions. FEMS Microbiol Lett 58, 221-225.

Gladysheva, T. B., Oden, K. L. \& Rosen, B. P. (1994). Properties of the arsenate reductase of plasmid R773. Biochemistry 33, 7288-7293.

Higgins, D. G. \& Sharp, P. M. (1988). Clustal: a package for performing multiple sequence alignment on a microcomputer. Gene 73, 237-244.

Higgins, D. G. \& Sharp, P. M. (1989). Fast and sensitive multiple sequence alignments on a microcomputer. Comput Appl Biosci 5, 151-153.

Huang, H., Huang, C. F., Wu, D. R., Jinn, C. M. \& Jan, K. Y. (1993). Glutathione as a cellular defence against arsenite toxicity in cultured Chinese hamster ovary cells. Toxicology 79, 195-204. 
Huckle, J. W., Morby, A. P., Turner, J. S. \& Robinson, N. J. (1993). Isolation of a prokaryotic metallothionein locus and analysis of transcriptional control by trace metal ions. Mol Microbiol 7, $177-187$.

Ji, G. \& Silver, S. (1992a). Reduction of arsenate to arsenite by the ArsC protein of the arsenic resistance operon of Staphylococcus aureus plasmid pI258. Proc Natl Acad Sci USA 89, 9474-9478.

Ji, G. \& Silver, S. (1992b). Regulation and expression of the arsenic resistance operon from Staphylococcus aureus plasmid pI258. J Bacteriol 174, 3684-3694.

Ji, G., Garber, E. A. E., Armes, L. G., Chen, C. M., Fuchs, J. A. \& Silver, S. (1994). Arsenate reductase of Staphylococcus aureus pl258. Biochemistry 33, 7294-7299.

Kaur, P. \& Rosen, B. P. (1992). Plasmid-encoded resistance to arsenic and antimony. Plasmid 27, 29-40.

Kaur, P. \& Rosen, B. P. (1994a). Identification of the site of $[\alpha-$ $\left.{ }^{32} \mathrm{P}\right]$ ATP adduct formation in the ArsA protein. Biochemistry 33, 6456-6461.

Kaur, P. \& Rosen, B. P. (1994b). In vitro assembly of an anionstimulated ATPase from peptide fragments. J Biol Chem 269, 9698-9704.

Li, J., Liu, S. \& Rosen, B. P. (1996). Interaction of ATP binding sites in the Ars A ATPase, the catalytic subunit of the Ars pump. J Biol Chem 271, 25247-25252.

Miller, J. H. (1972). Experiments in Molecular Genetics. Cold Spring Harbor, NY: Cold Spring Harbor Laboratory.

Minamishima, Y., Takeya, K., Ohnishi, Y. \& Amako, K. (1968). Physicochemical and biological properties of fibrous Pseudomonas bacteriophages. J Virol 2, 208-213.

Mobley, H. L. T. \& Rosen, B. P. (1982). Energetics of plasmidmediated arsenate resistance in Escherichia coli. Proc Natl Acad Sci USA 79, 6119-6122.

Myers, E. W. \& Miller, W. (1988). Optimal alignments in linear space. Comput Appl Biosci 4, 11-17.

Neyt, C., Iriarte, M., Thi, V. H. \& Cornelis, G. R. (1997). Virulence and arsenic resistance in Yersiniae. J Bacteriol 179, 612-619.

Oden, K. L., Gladysheva, T. B. \& Rosen, B. P. (1994). Arsenate reduction mediated by the plasmid-encoded ArsC protein is coupled to glutathione. Mol Microbiol 12, 301-306.

Owolabi, J. B. \& Rosen, B. P. (1990). Differential mRNA stability controls relative gene expression within the plasmid-encoded arsenical resistance operon. J Bacteriol 172, 2367-2371.

Rosen, B. P., Weigel, U., Karkaria, C. \& Gangola, P. (1988). Molecular characterization of an anion pump. The ars $A$ gene product is an arsenite (antimonate)-stimulated ATPase. J Biol Chem 263, 3067-3070.

Rosenstein, R., Peschel, P., Wieland, B. \& Gotz, F. (1992). Expression and regulation of the Staphylococcus xylosus antimonite, arsenite, and arsenate resistance operon. J Bacteriol 174, 3676-3683.

Sambrook, J., Fritsch, E. F. \& Maniatis, T. (1989). Molecular
Cloning: a Laboratory Manual, 2nd edn. Cold Spring Harbor, NY: Cold Spring Harbor Laboratory.

San Francisco, M. J. D., Hope, C. L., Owolabi, J. B., Tisa, L. S. \& Rosen, B. P. (1990). Identification of the metalloregulatory element of the plasmid-encoded arsenical resistance operon. Nucleic Acids Res 18, 619-624.

Silver, S. (1996). Bacterial resistances to toxic metal ions - a review. Gene 179, 9-19.

Silver, S., Ji, G., Bröer, S., Dey, S., Dou, D. \& Rosen, B. P. (1993). Orphan enzyme or patriarch of a new tribe: the arsenic resistance ATPase of bacterial plasmids. Mol Microbiol 8, 637-642.

Sofia, H. J., Burland, V., Daniels, D. L., Plunkett, G., III \& Blattner, F. R. (1994). Analysis of the Escherichia coli genome. V. DNA sequence of the region from 76.0 to 81.5 minutes. Nucleic Acids Res 22, 2576-2586.

Takeya, K. \& Amako, K. (1966). A rod-shaped Pseudomonas phage. Virology 28, 163-165.

Tisa, L. S. \& Rosen, B. P. (1990). Molecular characterization of an anion pump. The ArsB protein is the membrane anchor for the ArsA protein. J Biol Chem 265, 190-194.

Turner, R. J., Hou, Y., Weiner, J. H. \& Taylor, D. E. (1992). The arsenic ATPase efflux pump mediates tellurite resistance. $J$ Bacteriol 174, 3092-3094.

Vieira, J. \& Messing, J. (1987). Production of single-stranded plasmid DNA. Methods Enzymol 153, 3-11.

Wang, Z. \& Rossman, T. G. (1993). Stable and inducible arsenite resistance in Chinese hamster cells. Toxicol Appl Pharmacol 118, 80-86.

Wang, Z., Hou, G. \& Rossman, T. G. (1994). Induction of arsenite tolerance and thermotolerance by arsenite occurs by different mechanisms. Environ Health Perspect 102 (Suppl. 3), 97-100.

Wang, Z., Dey, S., Rosen, B. P. \& Rossman, T. G. (1996). Effluxmediated resistance to arsenicals in arsenic-resistant and -hypersensitive Chinese hamster cells. Toxicol Appl Pharmacol 137, 112-119.

West, S. E. H. \& Iglewski, B. H. (1988). Codon usage in Pseudomonas aeruginosa. Nucleic Acids Res 16, 9323-9335.

Wu, J. \& Rosen, B. P. (1991). The ArsR protein is a trans-acting regulatory protein. Mol Microbiol 5, 1331-1336.

Wu, J. \& Rosen, B. P. (1993a). Metalloregulated expression of the ars operon. J Biol Chem 268, 52-58.

Wu, J. \& Rosen, B. P. (1993b). The ars D gene encodes a second trans-acting regulatory protein of the plasmid-encoded arsenical resistance operon. Mol Microbiol 8, 615-623.

Wu, J., Tisa, L. S. \& Rosen, B. P. (1992). Membrane topology of the ArsB protein, the membrane subunit of an anion-translocating ATPase. J Biol Chem 267, 12570-12576.

Yanisch-Perron, C., Vieira, J. \& Messing, J. (1985). Improved M13 phage cloning vectors and host strains: nucleotide sequences of the M13mp18 and pUC19 vectors. Gene 33, 103-109.

Received 2 February 1998; revised 19 June 1998; accepted 29 June 1998. 